\title{
Multi-omics integrative analysis and survival risk model construction of non-small cell lung cancer based on The Cancer Genome Atlas datasets
}

\author{
MINGYUAN LUAN ${ }^{1}$, FUCHENG SONG ${ }^{2}$, SHUYUAN QU ${ }^{1}$, XI MENG $^{2}$, JUNJIE JI $^{1}$, \\ YUNBO DUAN $^{3}$, CHANGGANG SUN $^{4}$, HONGZONG SI $^{2,3}$ and HONGLIN ZHAI ${ }^{5}$ \\ ${ }^{1}$ School of Basic Medicine, Qingdao University Medical College; ${ }^{2}$ Department of Public Health, Qingdao \\ University Medical College; ${ }^{3}$ Institute for Computational Science and Engineering, Laboratory of New \\ Fibrous Materials and Modern Textile State Key Laboratory, Qingdao University, Qingdao, Shandong \\ 266071; ${ }^{4}$ Department of Cancer Center, Weifang Traditional Chinese Medicine Hospital, Weifang, Shandong \\ 262699; ${ }^{5}$ Department of Chemistry, Lanzhou University, Lanzhou, Gansu 730000, P.R. China
}

Received April 27, 2019; Accepted April 20, 2020

DOI: $10.3892 / \mathrm{ol} .2020 .11919$

\begin{abstract}
Lung cancer is a major cause of cancer-associated mortality worldwide. However, the association between multi-omics data and survival in lung cancer is not fully understood. The present study investigated the performance of the methylation survival risk model in multi-platform integrative molecular subtypes and aimed to identify copy number $(\mathrm{CN})$ variations and mutations that are associated with survival risk. The present study analyzed 439 lung adenocarcinoma cases based on DNA methylation, RNA, microRNA (miRNA), DNA copy number and mutations from The Cancer Genome Atlas datasets. First, six cancer subtypes were identified using integrating DNA methylation, RNA, miRNA and DNA copy number data. The least absolute shrinkage and selection operator (LASSO) regression algorithm was used to extract methylation sites of survival model and calculate the methylation-based survival risk indices for all patients. Survival for patients in the high-risk group was significantly lower compared with that for patients in the low-risk group $(\mathrm{P}<0.05)$. The present study also assessed methylation-based
\end{abstract}

Correspondence to: Professor Hongzong $\mathrm{Si}$, Institute for Computational Science and Engineering, Laboratory of New Fibrous Materials and Modern Textile State Key Laboratory, Qingdao University, 308 Ningxia Road, Qingdao, Shandong 266071, P.R. China

E-mail: sihz03@126.com

Abbreviations: LUAD, lung adenocarcinoma; NSCLC non-small cell lung cancer; TCGA, The Cancer Genome Atlas; c index, concordance index; OS, overall survival; AUC, area under the curve; $\mathrm{LOH}$, loss of heterozygosity; HRD, homologous repair deficiency; BIC, Bayesian Information Criterion

Key words: multi-omics, survival analysis, methylation, copy number, mutation, survival survival risks of the six subtypes and analyzed the association between survival risk and non-silent mutation rate, number of segments, fraction of segments altered, aneuploidy score, number of segments with loss of heterozygosity (LOH), fraction of segments with LOH and homologous repair deficiency. Finally, the specific copy number regions and mutant genes associated with the different subtypes were identified $(\mathrm{P}<0.01)$. Chromosome regions $17 \mathrm{q} 24.3$ and $11 \mathrm{p} 15.5$ were identified as those with the most survival risk-associated copy number variation regions, while a total of 29 mutant genes were significantly associated with survival $(\mathrm{P}<0.01)$.

\section{Introduction}

Currently, lung cancer is the most prevalent malignancy worldwide, with an estimated 1.76 million deaths in 2018 (1). Non-small cell lung cancer (NSCLC) accounts for $85 \%$ of primary lung cancer cases (2). The majority of patients with lung cancer are at an advanced stage when diagnosed, and the 5 -year survival rate is $<18 \%$ (3).

NSCLC is a highly complex and heterogeneous disease (4). Currently, molecular testing, often by next-generation sequencing, is routinely used at the time of diagnosis to guide treatment decisions for patients with NSCLC (1). Several genes mutations have been identified in patients with NSCLC, such as KRAS (25-35\%), EGFR (10\%), ALK (3-7\%), ROS1 (1-2\%) (5). Inhibition of these mutations through targeted small molecule drugs or antibody-based strategies has emerged as an effective approach to NSCLC therapy (6-8). However, a number of patients with NSCLC lack an identifiable driver oncogene, thus targeted therapies are not effective for these patients (2). The 5-year survival rate for these patients remain low (3). Thus, only mutation features cannot completely characterize the cancer genome or precisely pinpoint the cancer-driving mechanism. The Cancer Genome Atlas (TCGA) project proposed the concept of multi-platform integrative molecular subtyping in 2012 (9), which provides a paradigm for the discovery of novel cancer subtypes by integrating different omics data. Utilizing 
iCluster algorithm (10), TCGA Research Network divided patients into different subtypes by integrating different omics data. Each subtype is called iCluster, for example iCluster 1 , iCluster 2. By comparing the omics data in different iClusters, researchers can identify the distinctive omics features of each iCluster. In hepatocellular carcinoma, TCGA Research Network identified three iClusters for 196 patients (11). iCluster 2 and iCluster 3 exhibited a high frequency of $C D K N 2 A$ silencing by DNA hypermethylation and high frequency of mutations of TERT, CTNNBI and HNF1A. iCluster 1 had a low frequency of $C D K N 2 A$ silencing and low frequency of mutations of CTNNB1 and TERT. Furthermore, in lung adenocarcinoma (LUAD), TCGA Research Network also identified six iClusters for 230 patients (12). iClusters 1-3 frequently harbor TP53 mutations. Copy number associated gene expression changes on $3 \mathrm{q}$ in iCluster $1,8 \mathrm{q}$ in iCluster 2 , $15 q$ in iCluster 3, 6q in iCluster 4 and $19 p$ in iCluster 5 were observed. In summary, the different omics features of cancer can be characterized more accurately based on the multi-omics subtypes. Understanding the different characteristics of each multi-omics subtype may promote effective individualized therapy for lung cancer; however, further integrated analyses should be performed to investigate the associations between different omics features and to elucidate the association of these features with clinical features.

With respect to epigenetic alterations, DNA methylation serves an important role in cancer development. Yue et al (13) revealed that PTEN methylation inhibits cell apoptosis in NSCLC, while Shahabi et al (14) found that FOXA2 was hypermethylated in LUAD leading to low LINC00261 expression levels, and Yun et al (15) reported that overexpression of $I L-32 \gamma$ decreases lung tumor growth by inducing TIMP-3 hypomethylation. Hypermethylation of the $C A C N A 2 D 2$ promoter can promote proliferation and invasion of NSCLC (16). However, few studies have conducted methylation analysis of NSCLC from the perspective of a survival risk model and these models have been constructed based on mRNA profiles (17-19).

The present study primarily focused on the association between mRNA, methylation, miRNA, copy number variation, mutations and patient survival. The performance of the methylation survival risk model in multi-platform integrative molecular subtypes was investigated, while the aim was to also identify copy number variations and mutations, which were associated with survival risk.

\section{Materials and methods}

Multi-platform integrative clustering using iCluster. To reveal the subtypes formed by integrating various molecular platforms of NSCLC, the present study utilized the iCluster package (version 2.1.0) in R $(10,20)$. Clinical data and the data of four molecular platforms, including DNA copy number, DNA methylation, mRNA expression and microRNA (miRNA) expression levels of NSCLC, were downloaded from TCGA using TCGAbiolinks (21). Four molecular platforms were provided as input to iCluster: DNA copy number, DNA methylation, mRNA expression and miRNA expression. Copy number variation data were derived from circular binary segmentation data from the Affymetrix SNP 6.0 platform (Thermo Fisher Scientific, Inc.), and further decreased to a set of non-redundant regions as previously described (22). Probes in the methylation data (Illumina Infinium 450k arrays) with $>20 \%$ missing values were removed and methylation probes corresponding to SNP and sex chromosomes were also removed. In addition, for mRNA and miRNA data, probes with $>25 \%$ missing values were removed. mRNA and miRNA expression matrices were $\log 2$ transformed and normalized. The probes were then merged with the corresponding gene and their average value was selected as the gene expression level. The remaining probes of methylation data, mRNA and miRNA expression levels with no available values were uploaded into the CancerSubtypes (version 1.6.0) R package (23). The mRNA and miRNA set were filtered to remove genes with a standard deviation $<1.0$ across all tumor samples. DNA $\mathrm{CN}$, DNA methylation, mRNA and miRNA expression levels were subsequently uploaded into the iCluster algorithm. The optimal combination of clusters was determined using the Bayesian Information Criterion (BIC) method (24). The iCluster algorithm was used, with a different number of clusters (from 1 to 6). According to the iCluster algorithm manual, the heatmap of the outcome was compared with the different number of clusters to determine the optimal number of clusters based on the features pattern. Survival analysis of all subtypes was performed using the survminer (version 0.4.3) $\mathrm{R}$ package (25) and survMisc (version 0.5.5) R package (26). For patients in different iClusters, the Renyi test was used to compare patients' survival at the beginning, the middle and the end of the study, respectively. P-values between each two iClusters were calculated using the Renyi test. Patients without iCluster information were excluded from the survival analysis.

Constructing the DNA methylation prognosis prediction model. Patients with NSCLC, without definite survival time or survival status were screened out. The summary of patients' clinical data that were selected are presented in Table SI. The methylation data was used which removed probes with $>20 \%$ missing value or corresponding to SNP and sex chromosomes. The NA values were imputed using CancerSubtypes (version 1.6.0) R package (23), based on k-Nearest Neighbor algorithm. The preprocess Core R package (version 1.44.0) (27) was used to remove batch effects for the methylation dataset. Univariate Cox proportional risk regression model with the threshold of $\mathrm{P}<0.001$ was used to screen the methylation level of each $\mathrm{CpG}$ site associated with the overall survival (OS) of patients using the survival R package (version 2.44-1.1) (28). Least absolute shrinkage and selection operator (LASSO) regression was used to identity the most powerful methylation prognostic markers from survival related $\mathrm{CpG}$ sites using the glmnet R package (version 2.0-16) (29). Coefficients of certain $\mathrm{CpG}$ sites were reduced to zero by forcing the sum of the absolute value of the regression coefficients to be less than a fixed value. The following steps were repeated ten thousand times: (1) Patients were randomly divided into training set and test set. The training set was composed of $70 \%$ patients and the test set was composed of $30 \%$ patients. (2) The methylation prognostic model was assessed on the on the training set and the performance of the model was assessed on the test set. (3) Utilizing Hmisc package (version 4.4-0) (30) in R, the concordance index (c index) of prognostic model in the training set and the test set were calculated, respectively. Subsequently, 
the performance score of the model was calculated using the following formula: performance score $=\mathrm{c}$ index $(\text { training set })^{2}+$ c index(test set $)^{2}$.

The risk score for each patient was calculated by combining the relative methylation levels of the prognostic CpG sites and LASSO coefficients. Multivariate Cox regression analysis was performed for the survival data to determine if the methylation score was independently predictive of survival. The performance of methylation score was assessed using time-dependent survival ROC curve. The present study identified the optimal cut-off risk score based on the optimum sensitivity and specificity of the 5-year survival ROC curve. Patients were divided into high- and low-risk groups according to the optimal cut-off score (-11.707). Kaplan-Meier survival analysis was used to estimate the survival of patients between the high- and low-risk groups. The correlation between all $\mathrm{CpG}$ sites and risk score, all mRNA and risk scores and all miRNA and risk scores were calculated using the cor.test function in R. P-values were generated using a two-sided t-test. According to the correlations and P-values, the most closely associated CpG sites, mRNA and miRNA were selected. Bonferroni's method was used to adjust the significance level of multiple comparisons, using the following formula:

$$
\alpha^{\prime}=\frac{\alpha}{k(k-1) / 2}
$$

Survival risk associations between somatic variation and germline variation. All loss of heterozygosity (LOH), aneuploidy, homologous repair deficiency (HRD) scores, copy number burden and mutation burden used in the present study were derived from the Genomic Data Commons website (https://gdc.cancer.gov/node/998) (31). Copy number burden scores are for fractions of segments altered and the number of segments. These two scores represent the fraction of bases deviating from baseline ploidy (defined as $>0.1$ or $<-0.1$ in $\log 2$ relative copy number space), and the total number of segments in the copy number profile of each sample. $\mathrm{LOH}$ means the irreversible loss of one of the parental alleles. The number of segments with $\mathrm{LOH}$ represents the number of segments with $\mathrm{LOH}$ events. The fraction of segments with $\mathrm{LOH}$ refers to the fraction of bases with LOH events. HRD score measures defects in homologous recombination. HRD score is the sum of three separate metrics of genomic scarring: Large $(>15 \mathrm{Mb})$ non-arm-level regions with $\mathrm{LOH}$, large-scale state transitions (breaks between adjacent segments of $>10 \mathrm{Mb}$ ) and subtelomeric regions with allelic imbalance. Aneuploidy scores were calculated as the sum total of altered (amplified or deleted) arms (32).

Copy number variation data, which uses GISTIC 2.0 (http://software.broadinstitute.org/cancer/cga/gistic) to define amplification and deletion, were downloaded from cBioPortal $(33,34)$. To identify the effects of genes copy number amplification and deletion on survival risk, the significant differences between the risk scores of patients with and without copy number variation were assessed. For each gene, the risk scores between patients with gene amplification and patients without gene alterations were compared. Subsequently, the risk scores between patients with gene deletions and patients without gene alterations were compared. Welch's two-sample t-tests were used to assess significant differences. The present study then analyzed whether the mRNA level of these survival-associated genes can be influenced by copy number amplification or deletion using Kruskal-Wallis tests in ggpubr package (version 0.2) (35) in R. All P-values to compare differences between two groups were calculated using Wilcoxon rank sum tests. Bonferroni's method was used to adjust the significance level of multiple comparisons, using the following formula:

$$
\alpha^{\prime}=\frac{\alpha}{k(k-1) / 2}
$$

Mutation data were downloaded from cBioPortal. A two-sample t-test was used to screen genes in which mutations were significantly associated with risk score. Genes with $\mathrm{P}<0.01$ were selected. The co-occurrence and exclusivity of risk-related genes were compared using maftools $\mathrm{R}$ package (version 1.6.15) (36). P-values were calculated using pair-wise Fisher's exact tests. P-values were adjusted using the FDR method.

\section{Results}

Multi-platform integrative molecular subtyping. A total of 423 patients had complete omics data, thus iCluster algorithm analysis was performed on these patients. An overview of the analytical strategy is shown in Fig. S1. Joint multivariate regression of the four platforms (DNA copy number, DNA methylation, mRNA expression and miRNA expression) calculated the BIC from two iClusters to six iClusters (Fig. S2). The BIC of the different number of iClusters did not show an inflection point, suggesting that the data generated were noisy. According to the of iCluster algorithm manual, the heatmaps from two iClusters to six iClusters were compared (Fig. S3). We considered that six iClusters can reflect the features of different omics data most detailed. Thus, we selected six the optimal number of iClusters. Subsequently, the features of omics data in different iClusters were analyzed (Fig. 1 and Table SII). Among the six iClusters, copy number variation is the most variable omics data while miRNA converse. iCluster $1(n=36)$, was characterized by a high frequency of KEAP1 mutations (63.89\%) and low frequency of TP53 mutations $(11.11 \%)$. iCluster $2(n=94)$, iCluster $4(n=50)$, iCluster $5(n=80)$ and iCluster $6(n=68)$ were characterized by high frequencies of TP53 mutations $(75.53,64,68.75$ and $66.18 \%$, respectively). iCluster $6(n=68)$ was characterized by clinical associations with higher AJCC stage (37). iCluster 1 and iCluster 6 had more male patients, while iCluster 3 and iCluster 5 were reversed. TTN mutations were observed in iCluster 2 , iCluster 4 , iCluster 5 and iCluster $6(56.9,70,57.5$ and $67.1 \%$ respectively). The distribution of TTN mutations was similar to TP53. iCluster 3 was associated with $30.5 \%$ KRAS mutations, which was the most frequent mutation in this molecular subtype. Multi-platform integrative molecular subtyping was associated with OS (Fig. 2). A total of 27 patients without iCluster information were excluded from the survival analysis. The Renyi test demonstrated significant differences for the beginning $(\mathrm{P}<0.001)$ and middle of the study $(\mathrm{P}<0.05)$ but not for the later stage $(P>0.1)$. Survival analysis was implemented 


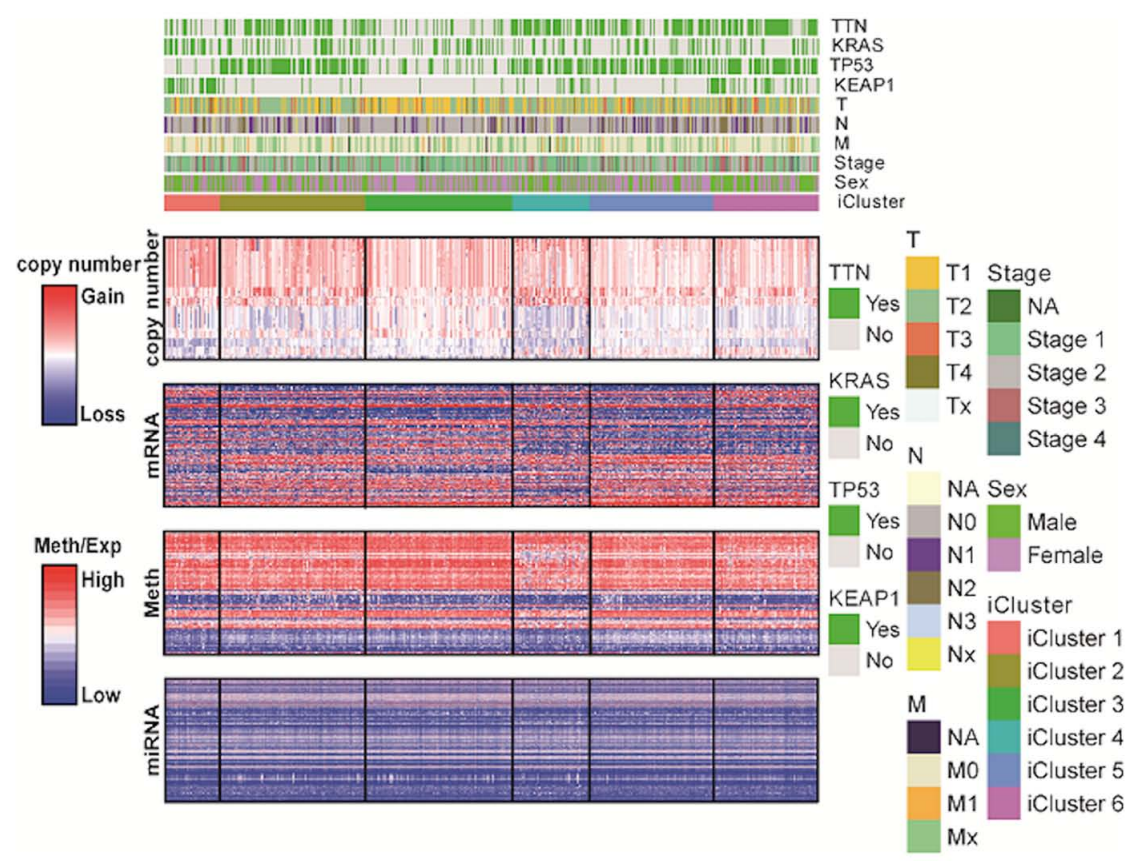

Figure 1. Multi-platform clustering analysis identified six integrated molecular subtypes of non-small cell lung cancer. Heatmaps organized by iCluster groupings for DNA CN, mRNA expression levels, DNA methylation levels and miRNA expression levels and their associations with selected mutations and clinical features (top tracks). Patients are in columns, grouped by the iCluster membership. Meth, methylation; Exp, expression; T, tumor; N, node; M, metastasis; NA, not available.

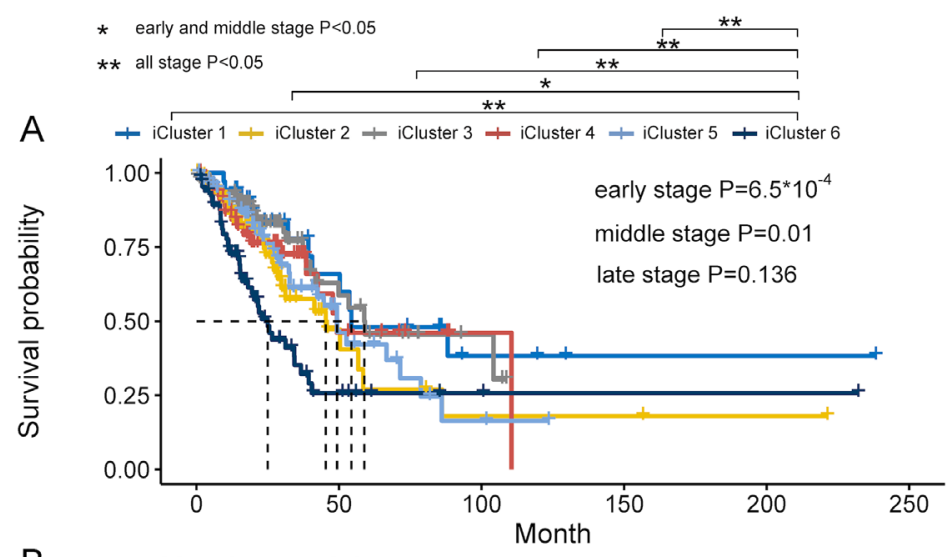

B Number at risk
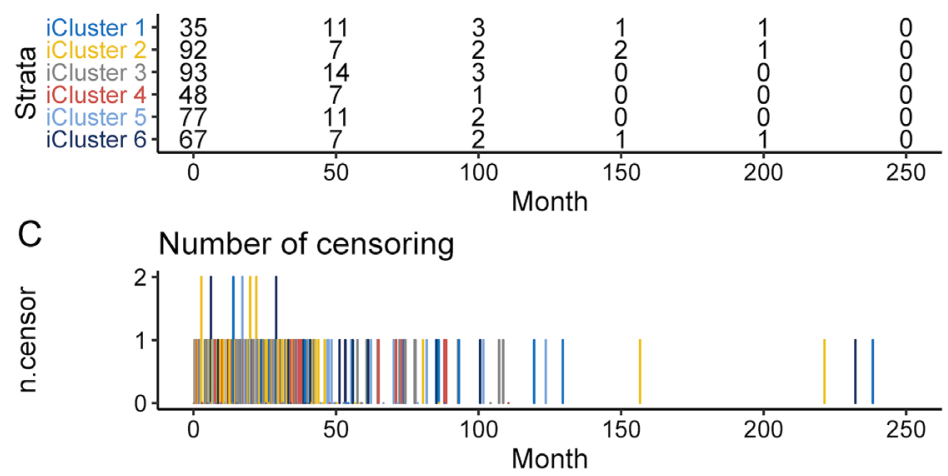

Figure 2. Survival analysis of the six integrated molecular subtypes of non-small cell lung cancer. (A) The outcomes of survival analysis. iCluster 6 had the least favorable outcome for overall survival, while iCluster 1 ,iCluster 2 , iCluster 3 , iCluster 4 and iCluster 5 had the better prognosis compared with iCluster 6 . Statistically significant differences were observed for the beginning $(\mathrm{P}<0.001)$ and middle of the study $(\mathrm{P}<0.05)$ but not for the later stage $(\mathrm{P}>0.1)$. (B) The number of patients at risk in different months for iClusters 1-6. (C) The number of patient deaths in different times for iClusters 1-6. ${ }^{*} \mathrm{P}<0.05$, ${ }^{* *} \mathrm{P}<0.01$. n.censor, number cencored.

for a total of 412 patients. Patients had better prognosis in iCluster 1 , iCluster 3 , iCluster 4 and iCluster 5 compared with iCluster 6. For different iClusters, the number of patients at risk in different times are presented in Fig. 2B. For different iClusters, the number of patient deaths in different times are presented in Fig. 2C. 
Table I. Overall, $21 \mathrm{CpG}$ sites were selected as the most powerful prognostic markers using LASSO regression in patients with non-small cell lung cancer.

\begin{tabular}{|c|c|c|c|c|c|}
\hline \multirow[b]{2}{*}{ CpG site } & \multirow[b]{2}{*}{ Position } & \multicolumn{3}{|c|}{ Univariate cox regression analysis } & \multirow{2}{*}{$\begin{array}{c}\text { LASSO } \\
\text { coefficient }\end{array}$} \\
\hline & & HR & $95 \% \mathrm{CI}$ & P-value & \\
\hline $\operatorname{cg} 01467592$ & chr8: $144424051-144424331$ & 0.02 & $0.01-0.08$ & $<10^{-16}$ & -0.23727154 \\
\hline $\operatorname{cg} 02967813$ & chr2: $3635439-3635700$ & 0.04 & $0.01-0.23$ & $3 \times 10^{-4}$ & -1.215714791 \\
\hline $\operatorname{cg} 04391569$ & chr10: $132781789-132782190$ & 0.01 & $0-0.04$ & $<10^{-16}$ & -1.105381717 \\
\hline $\operatorname{cg} 05406101$ & chr21: $29018943-29019437$ & 0.14 & $0.05-0.37$ & $1.2 \times 10^{-4}$ & -0.527102271 \\
\hline $\operatorname{cg} 06860998$ & chr4: 4385866-4388192 & 0.03 & $0.01-0.21$ & $3.3 \times 10^{-4}$ & -1.509305485 \\
\hline $\operatorname{cg} 06933711$ & chr10: 30058670-30059719 & 0.03 & $0.01-0.11$ & $<10^{-16}$ & -0.966075621 \\
\hline $\operatorname{cg} 12193943$ & chr12: 1796111-1797599 & 0.02 & $0-0.07$ & $<10^{-16}$ & -0.305762883 \\
\hline $\operatorname{cg} 13372811$ & chr1: 110083906-110084862 & 6.43 & $2.2-18.77$ & $6.7 \times 10^{-4}$ & 0.524860897 \\
\hline $\operatorname{cg} 19160958$ & chr17: $37406724-37406967$ & 0.04 & $0.01-0.15$ & $<10^{-16}$ & -0.469969803 \\
\hline $\operatorname{cg} 21749275$ & chr4: $109559671-109560904$ & 0.03 & $0-0.21$ & $4 \times 10^{-4}$ & -1.227936552 \\
\hline $\operatorname{cg} 22697853$ & chr1: $148264740-148265029$ & 0.06 & $0.01-0.22$ & $4 \times 10^{-5}$ & -0.065212053 \\
\hline $\operatorname{cg} 27018309$ & chr16: $8868332-8869080$ & 0.01 & $0-0.03$ & $<10^{-16}$ & -1.016454431 \\
\hline $\operatorname{cg} 27529004$ & chr2: $236567668-236568620$ & 0.02 & $0-0.1$ & $1 \times 10^{-5}$ & -0.319014839 \\
\hline $\operatorname{cg} 00278107$ & chr5: 1060924-1061607 & 0 & $0-0.01$ & $1 \times 10^{-5}$ & -0.283330883 \\
\hline $\operatorname{cg} 03723506$ & chr5: 38556120-38557461 & 0 & $0-0.01$ & $8.9 \times 10^{-4}$ & -1.663229849 \\
\hline $\operatorname{cg} 04973915$ & chr5: $172006000-172007078$ & 0 & $0-0.01$ & $<10^{-16}$ & -0.286595288 \\
\hline $\operatorname{cg} 06720244$ & chr13: 46052319-46052884 & 0 & $0-0.02$ & $6.7 \times 10^{-4}$ & -3.055164476 \\
\hline $\operatorname{cg} 11302293$ & chr4: $3772124-3772335$ & 0 & $0-0.08$ & $5.2 \times 10^{-4}$ & -0.045241836 \\
\hline $\operatorname{cg} 13354228$ & chr18: $657401-658745$ & 0 & $0-0$ & $2 \times 10^{-5}$ & -8.258933249 \\
\hline $\operatorname{cg} 17510645$ & chr1: $243254439-243255473$ & 0 & $0-0$ & $<10^{-16}$ & -0.017156936 \\
\hline $\operatorname{cg} 20981791$ & chr8: $23288015-23288493$ & 0 & $0-0.08$ & $4.4 \times 10^{-4}$ & -1.539966176 \\
\hline
\end{tabular}

CI, confidence interval; HR, hazards ratio; chr, chromosome; LASSO, least absolute shrinkage and selection operator.

Constructing the DNA methylation prognosis prediction model. A total of 439 patients with survival times and survival status were selected to construct the DNA methylation prognosis prediction model. First, the present study removed the probes in the methylation data with $>20 \%$ missing values. The probes corresponding to SNP and sex chromosomes were also removed. After the pre-processing steps, univariate Cox proportional risk regression model analyses were performed with the threshold of $\mathrm{P}<0.001$ on the DNA methylation dataset. A total of $4,534 \mathrm{CpG}$ sites associated with OS were initially identified.

The relative regression coefficients of survival-associated $\mathrm{CpG}$ sites were then calculated using a LASSO analysis. Coefficients of certain $\mathrm{CpG}$ sites were reduced to zero by forcing the sum of the absolute value of the regression coefficients to be less than a fixed value. A total of $21 \mathrm{CpG}$ sites were selected as the most powerful prognostic marker (Table I). The detailed information of these $21 \mathrm{CpG}$ sites are presented in Table SIII.

The risk score for each patient was calculated by combining the methylation levels of the prognostic $\mathrm{CpG}$ sites with the corresponding LASSO coefficients. The outcome of multivariate Cox regression analysis for the survival data showed that the methylation score independently predicted survival (Table SIV). Cross-validated time-dependent ROC curves demonstrated that the 12 months AUC was 0.809 (Fig. 3B).
Both AUC of 36 months and AUC of 60 months were more $>0.7$. These reflect that our model had good ability of prognosis prediction in short term and long term. Patients were divided into high- and low-risk groups based on the cut-off value, which was generated according to the optimum sensitivity and specificity of the 5-year survival ROC curve. Patients with a risk score $\geq-11.707$ were assigned to the high-risk group, and the remaining patients into the low-risk group (Fig. 4A). The number of deaths in the high-risk group was significantly greater compared with that in the low-risk group (Fig. 4B). The survival time of patients in the low-risk score group was significantly longer compared with that of patients in the high-risk group $(\mathrm{P}<0.001$; Fig. 3A). As presented in Fig. 4C, the majority of these $21 \mathrm{CpG}$ sites in patients in the high-risk group exhibited low methylation levels.

The correlations between all $\mathrm{CpG}$ sites and risk score, all mRNAs and risk score, and all miRNAs and risk score were calculated using the cor.test function in R. Next, we screened the most related $\mathrm{CpG}$ sites, mRNA and miRNA based on correlation scores. High-risk scores were most significantly associated with higher methylation levels of ST6GALNAC6 (cg13939204) (Fig. 5A), as well as higher ANLN expression (Fig. 5B) and lower $h s a-m i R-29 c$ expression levels (Fig. 5C) in methylation, mRNA and miRNA, respectively. In addition, a similar significant association between risk score and stage was observed 




B

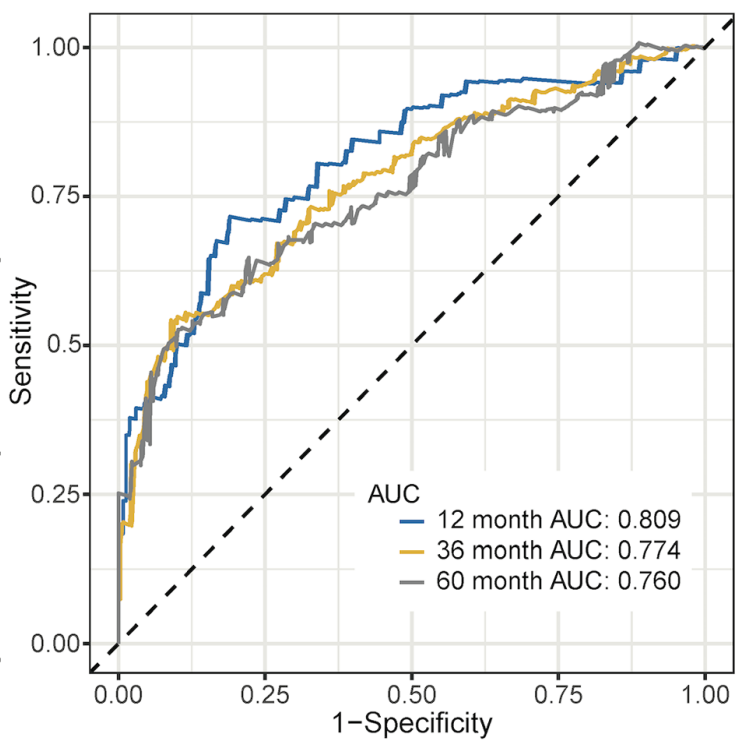

Figure 3. Performance of methylation survival model. (A) Kaplan-Meier survival analysis of the high- and low-risk groups. Patients in the low-risk group showed improved survival compared with those in the high-risk group. (B) The time-dependent ROC curves of methylation prognostic classifier of non-small cell lung cancer. AUCs at 1,3, and 5 years were used to assess prognostic accuracy. ROC, receiver operating characteristic; AUC, area under the curve; n.censor, number censored.

(Fig. S4A). Patients in stage 2 and stage 3 had higher risk scores compared with that in patients in stage 1 . The significance level was adjusted using Bonferroni's correction $\left(\alpha^{\prime}=8.3 \times 10^{-3}\right)$. The $\mathrm{P}$-values of comparisons between each two group are displayed in Fig. S4A. The mutation of TP53 was significantly associated with high risk (Fig. 5D). Risk scores in different molecular subtypes were significantly different (Fig. S4B). Risk scores in iCluster 4, iCluster 5 and iCluster 6 were significantly higher than iCluster 3 . Risk scores in iCluster 6 were significantly higher than iCluster 1 , iCluster 2, iCluster 3 and iCluster 5 . The significance level was adjusted using Bonferroni's correction $\left(\alpha^{\prime}=3.3 \times 10^{-3}\right)$. The P-values of comparisons between each two group are displayed in Fig. S4.

Survival risk associations between somatic variation and germline variation. Risk scores were positively correlated with non-silent mutation rate with the strongest correlation in iCluster 1. Risk scores were associated with copy number variation burden (number of segments and fraction of genome alterations) in iCluster 5 and iCluster 6 (Fig. 6A). Risk scores were also positively correlated with the number of segments with LOH in iCluster 5 and iCluster 6. In iCluster 2 and iCluster 6 , risk scores were positively correlated with the fraction of segments with LOH. Risk scores were positively correlated with homologous repair deficiency scores in iClsuter 1 and iCluster 5. These measures (number of segments, fraction of segments altered, number of segments with $\mathrm{LOH}$ and homologous repair deficiency) represented smaller focal copy number events and DNA damage. These results suggested that the alteration of focal copy number may increase patients' survival risk scores in iCluster 5 and iCluster 6 . High number or fraction of $\mathrm{LOH}$ events may lead high risk scores for patients in iCluster 2, iCluster 5 and iCluster 6. Furthermore, high homologous repair deficiency scores may lead high risk scores for patients in iCluster 1 and iCluster 5 .
Welch's two-sample t-tests were used to investigate the association between copy number variation and risk scores $(\mathrm{P}<0.01$; Fig. 6B). The red bars represent amplified regions and the blue bars indicate deletions. The darker bar color represents smaller P-values of the corresponding regions. The present study identified 35 copy number amplification regions (2p23, 2p24, 2p25, 3q25, 3q26, 3q27, 3q28, 3q29, 4p12, 7p11, 7p12, 7p13, 7q11, 10p15, 10q26, 12p11, 12p12, 12p13, 12q12, $12 \mathrm{q} 13,12 \mathrm{q} 14,12 \mathrm{q} 21,15 \mathrm{q} 23,15 \mathrm{q} 24,15 \mathrm{q} 25,15 \mathrm{q} 26,17 \mathrm{q} 11,17 \mathrm{q} 12$, $17 \mathrm{q} 21,17 \mathrm{q} 22,17 \mathrm{q} 23,17 \mathrm{q} 24,17 \mathrm{q} 25,19 \mathrm{q} 13$ and $22 \mathrm{q} 13)$ and 40 copy number deletion regions $(1 \mathrm{p} 34,1 \mathrm{p} 36,2 \mathrm{p} 11,2 \mathrm{q} 11,5 \mathrm{p} 12$, 5q11, 8p21, 9p11, 9p12, 9p13, 9p21, 9p22, 9p23, 9p24, 9q12, 9q13, 9q21, 11p11, 11p12, 11p13, 11p14, 11p15, 11q14, 11q21, 11q22, 11q24, 11q25, 14q22, 14q23, 14q24, 14q31, 14q32, $16 \mathrm{p} 13,18 \mathrm{q} 11,18 \mathrm{q} 12,19 \mathrm{p} 12,19 \mathrm{p} 13,19 \mathrm{q} 13,20 \mathrm{p} 12$ and 22q13) which were significantly associated with higher risk scores.. 17q24.3 amplification (including AC005152.3, LINC01152, RP11-84E24.2, SOX9-AS1, AC007461.2, SOX9, KCNJ16 and $S L C 39 A 11)$ and chromosome 11p15.5 deletion (MOB2, AP2A2, MUC6, MUC2, MUC5B, MIR6744, TOLLIP and TOLLIP-AS1) were most significantly associated with higher risk scores. Amplification of AC005152.3 was most correlated with higher risk scores in 17q24.3 (Fig. 6C and D). Deletion of $M O B 2$ was most correlated with higher risk scores in 11p15.5. Associations between amplification/deletion and the expression levels of genes in 17q24.3 and 11p15.5, demonstrating that six deletion genes (AP2A2, MOB2, MUC5B, MUC6, TOLLIP and TOLLIP-AS1) significantly influenced the levels of mRNA (Fig. 7), whereas two amplification (KCNJ16 and SLC39A11) genes affected the levels of mRNA (Fig. 8).

A total of 29 mutant genes were found to be significantly associated with risk scores $(\mathrm{P}<0.01$; Table II) and TP53 was most significantly correlated with risk score. Utilizing maftools $\mathrm{R}$ packages, pair-wise Fisher's exact tests were 

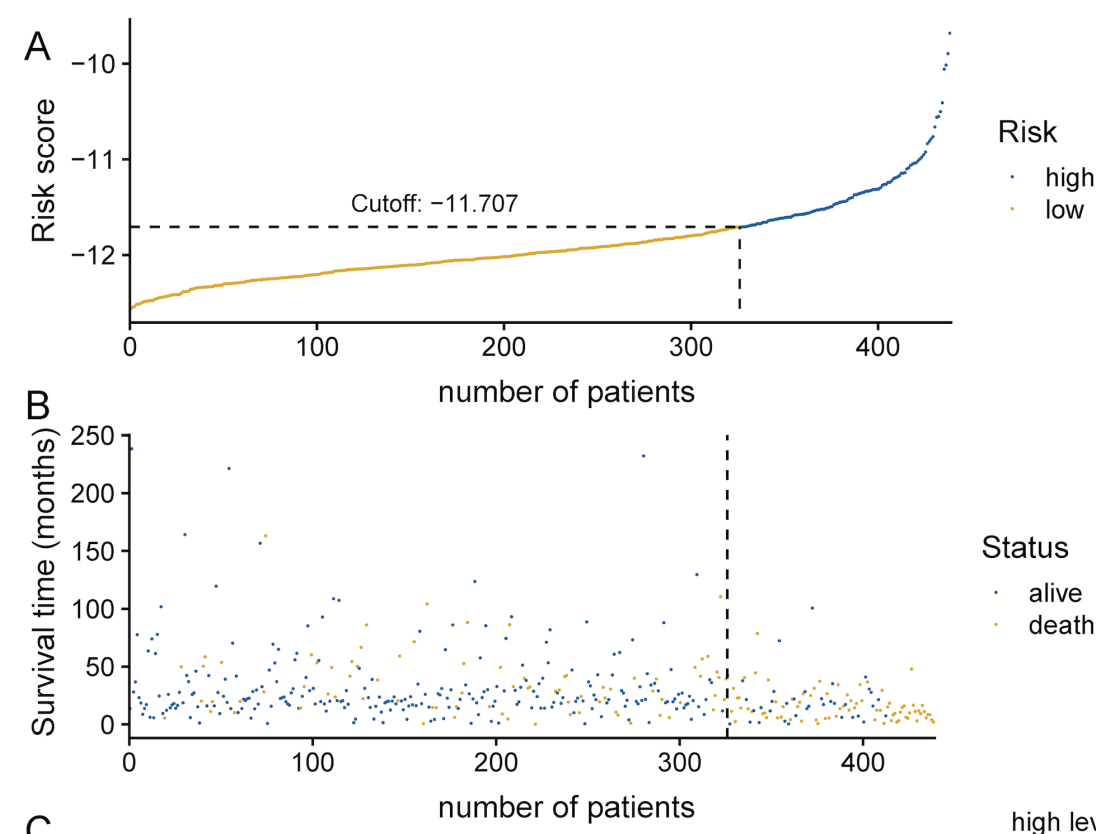

Status

alive

death

C

number of patients

high level

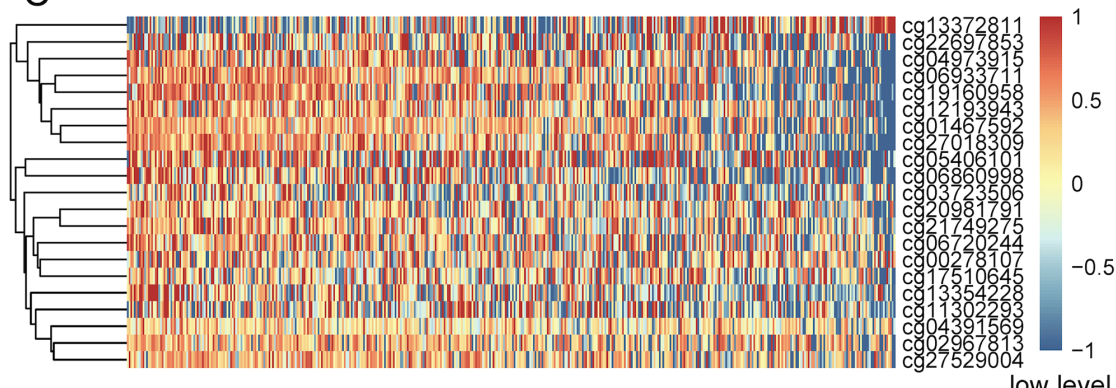

low level

Figure 4. Methylation prognostic classifier of non-small cell lung cancer. (A) The distribution of risk scores. The present study selected-11.7070 as the optimal cut-off value. Patients were divided into a high-risk (blue) and low-risk groups (yellow). (B) Patient survival time and status. The black dotted line represents the optimum cut-off dividing patients into low-risk (left) and high-risk (right) groups. The yellow dots represent death and the blue dot represents alive status. The number of deaths in the high-risk group was significantly greater compared with that in the low-risk group. (C) Heatmap of the methylation level of the $21 \mathrm{CpG}$ sites in the prognostic classifier. Red represents high level of methylation, while blue represents low level of methylation. The majority of $21 \mathrm{CpG}$ sites exhibited lower levels of methylation in the high-risk group compared with that in the low-risk group.

A

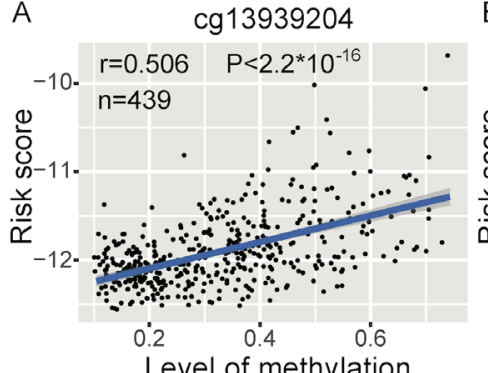

Level of methylation

C

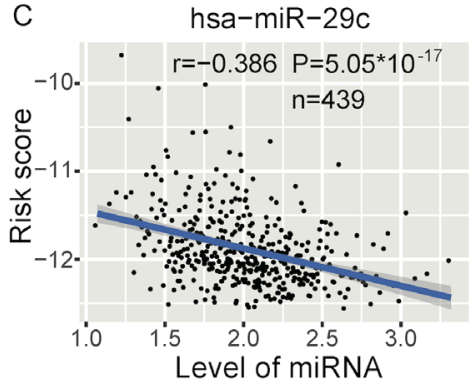

B

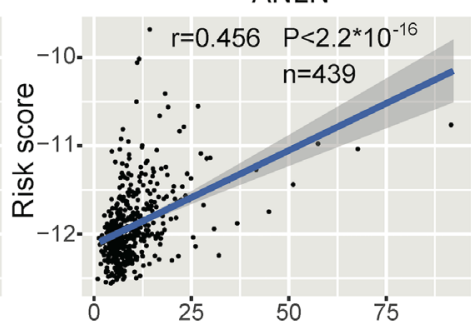

Level of mRNA
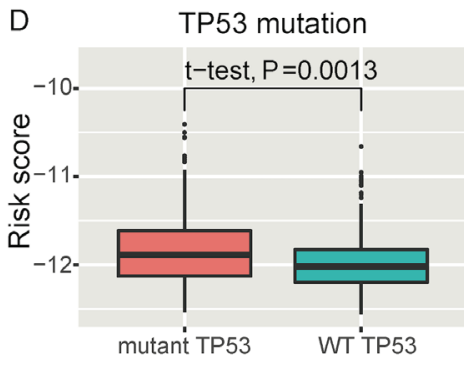

Figure 5. Molecular and clinical features associated with survival risk score in non-small cell lung cancer. Correlation scores of survival risk scores and (A) the most survival risk-associated CpG site (cg13939204), (B) the most survival risk-associated gene (ANLN) and (C) the most survival risk-associated miRNA (hsa-miR-29c). (D) The risk score of patients with and without TP53 mutation. Patients with the TP53 mutation had higher risk scores compared with patients with WT TP53. miRNA/miR, microRNA, WT, wild-type. 
A

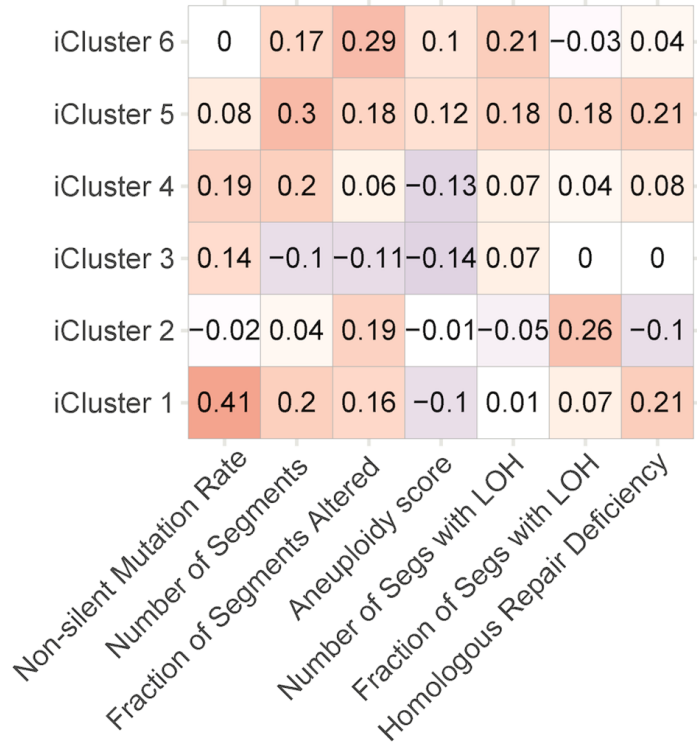

C

AC005152.3 追 Amplification 追 Deletion 追 Normal

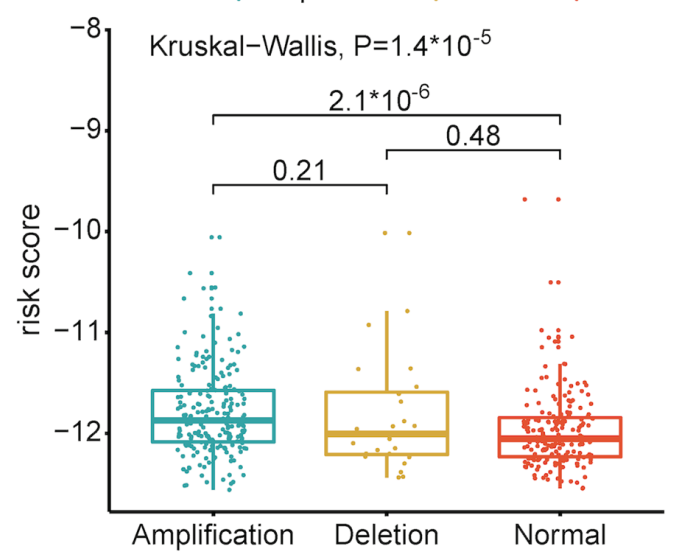

B
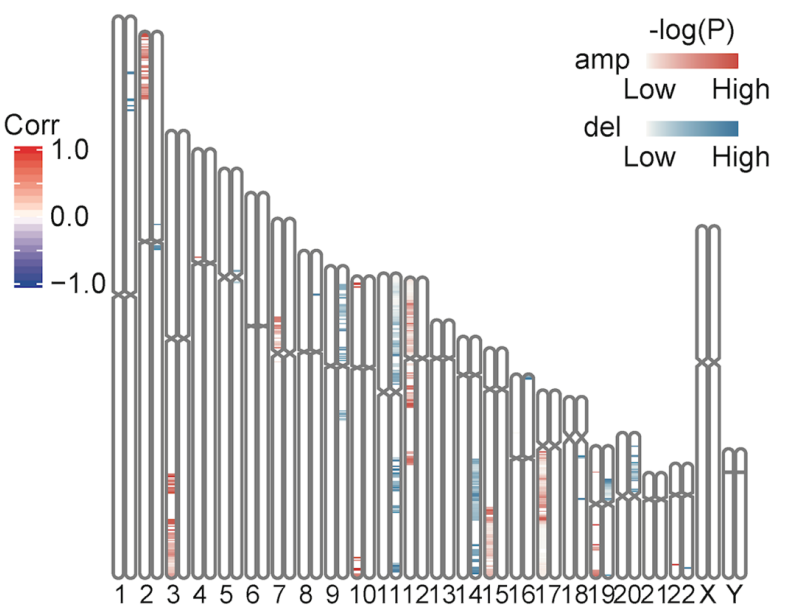

D

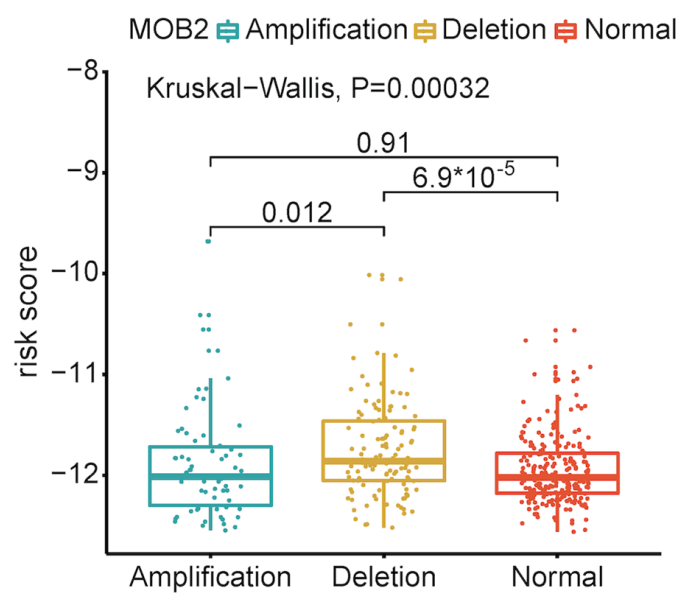

Figure 6. Association between copy number variation and survival risk score. (A) Association between DNA damage measures and survival risk scores in different molecular subtypes. (B) Copy number variation regions that were significantly associated with survival risk scores (P<0.01). (C) The risk scores of patients with different copy number variation statuses of AC005152.3 in chromosome region 17q24.3. The significance level was adjusted using Bonferroni's correction $\left(\alpha^{\prime}=0.0167\right)$. (D) Risk scores of patients with different copy number variation statuses of $M O B 2$ in chromosome region 11 p15.5. The significance level was adjusted using Bonferroni's correction $\left(\alpha^{\prime}=0.0167\right)$. Corr, correlation; amp, amplification; del, deletion; LOH, loss of heterozygosity.

used to identify the co-occurrence and exclusivity of genes screened in the present study. We identified that MUC16 significantly co-occurred with $H Y D I N, A P O B, R Y R 3$ and $L R P 1 B$ (FDR $<0.01$ and co-occurrence $\geq 3$; Fig. 9). $A P O B$ significantly co-occurred with TRPS1, MUC16, COL11A1, $C O L 22 A 1$ and $L R P 1 B($ FDR $<0.01$ and co-occurrence $\geq 3)$. $C S M D 3$ significantly co-occurred with $C O L 11 A 1, L R P 1 B$ and $T P 53$ (FDR $<0.01$ and co-occurrence $\geq 3$ ). ERBB4 significantly co-occurred with NLRP3 (FDR $<0.01$ and co-occurrence $\geq 3$ ). COL11A1 significantly co-occurred with $A P O B, C S M D 3$ and $C O L 22 A 1$ (FDR $<0.01$ and co-occurrence $\geq 3)$.

\section{Discussion}

In 2014, TCGA Research Network reported research regarding the multi-omics integrated analysis of LUAD (38).
TCGA Research Network identified that EGFR mutations were more frequent in women, and $R B M 10$ mutations were more common in men. A total of $4 \%$ of cases harbored exon 14 skipping in MET mRNA. Mutations in NF1, MET, ERBB2 and RIT1 may play driver roles in LUAD. TCGA Research Network inferred that phosphorylation of proteins may lead aberrant activation of $M A P K$ and $P I(3) K$ pathway. TCGA Research Network identified six integrated multi-omics $(\mathrm{CN}$, DNA methylation and mRNA expression data) subtypes using iCluster analysis. Meanwhile, the present study also identified six molecular subtypes based on integrated analysis of multi-omics data (copy number variation, mRNA, methylation and miRNA) and constructed a survival risk model based on NSCLC data. The present study investigated the role of methylation, copy number variation and mutation in the survival of patients with NSCLC. The data type of 
A

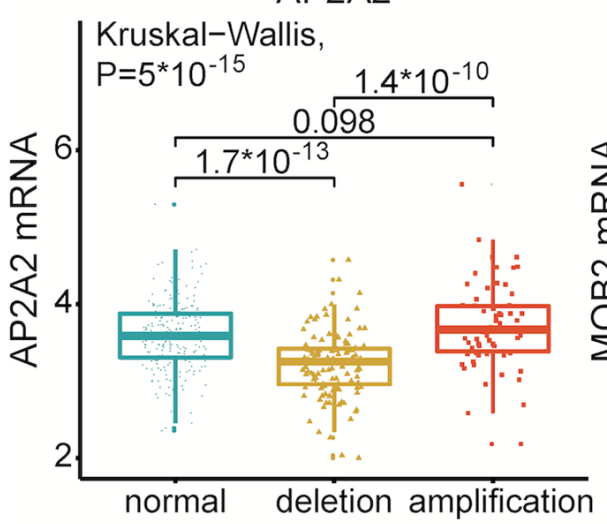

B

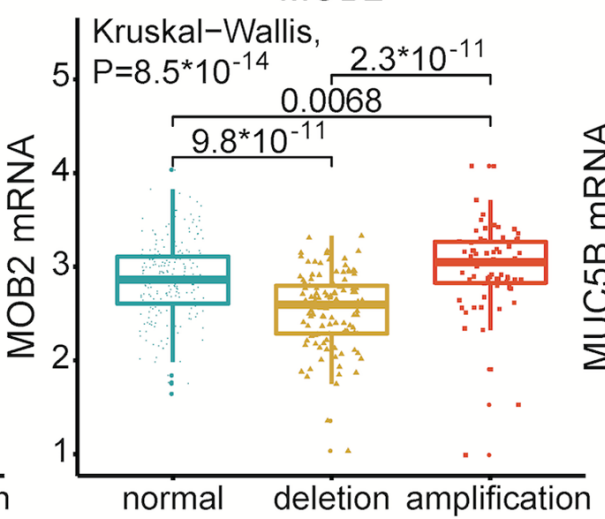

C MUC5B

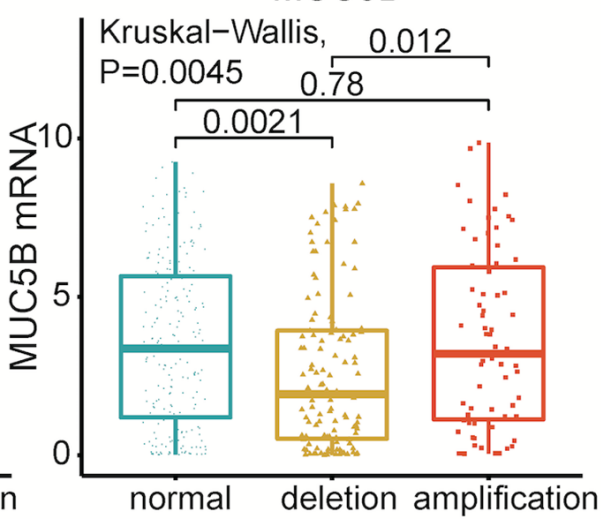

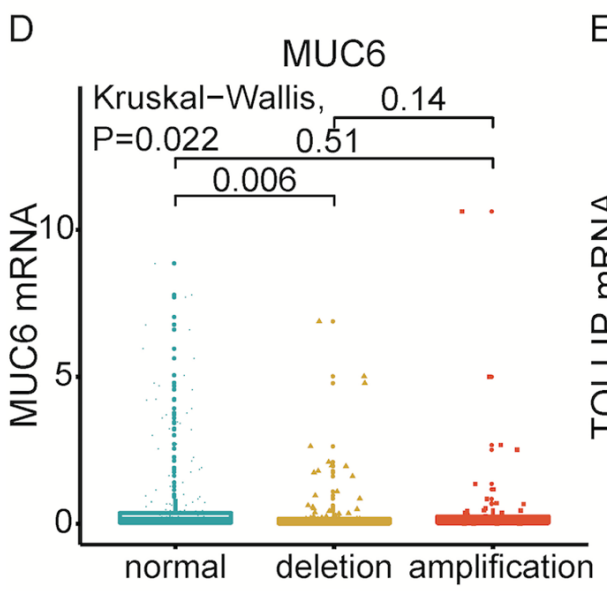

E

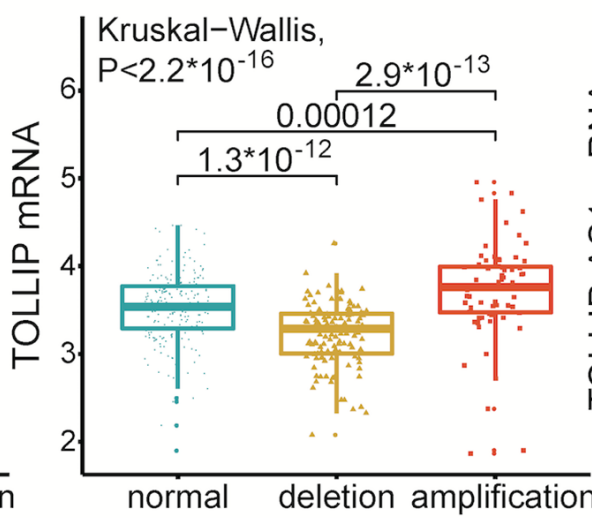

F

TOLLIP-AS1

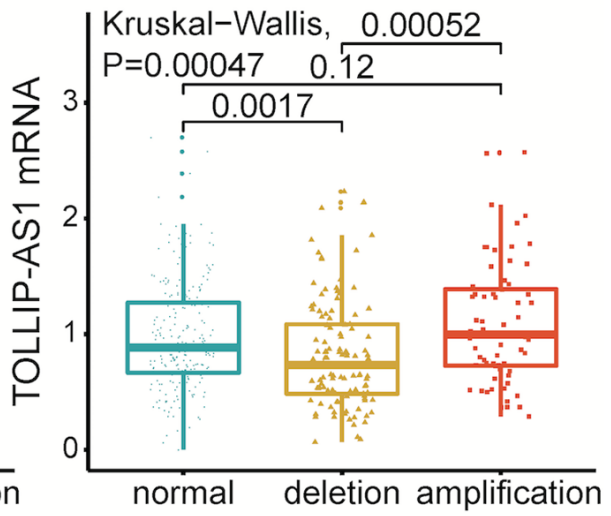

Figure 7. Survival-risk associated genes in which mRNA expression level is significantly associated with copy number variation deletions. mRNA expression levels of (A) AP2A2, (B) MOB2, (C) MUC5B, (D) MUC6, (E) TOLLIP and (F) TOLLIP-AS1 in different copy number variation status. Multiple comparisons were corrected using Bonferroni's method $\left(\alpha^{\prime}=0.0167\right)$. $\mathrm{CN}$, copy number.

A

KCNJ16 cnv 追normal 官 deletion 追amplification

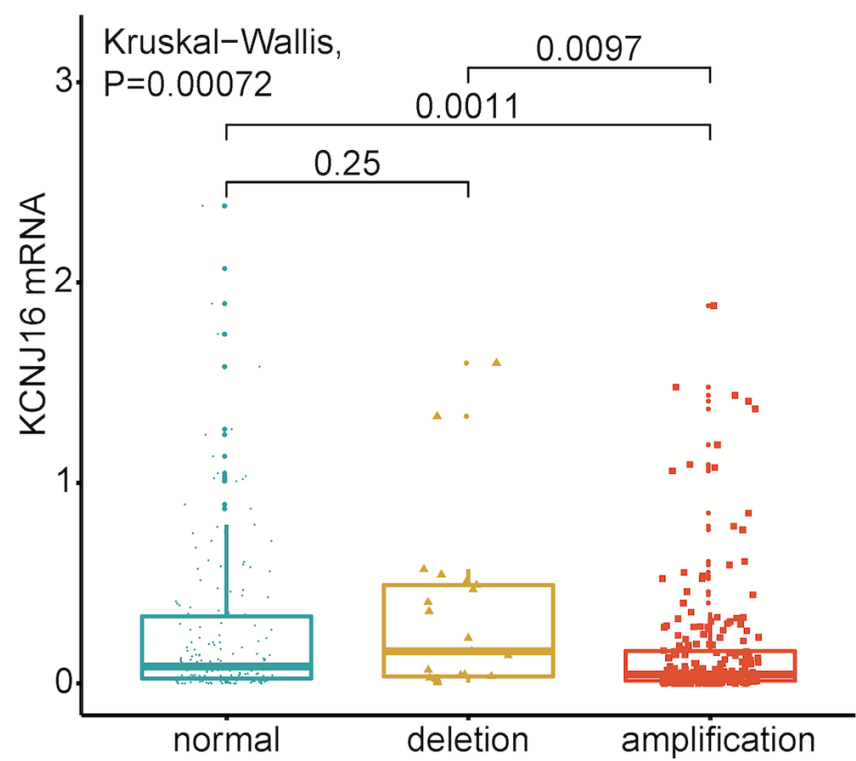

$\mathrm{B}$

SLC39A11 cnv 追normal 追 deletion 追amplification

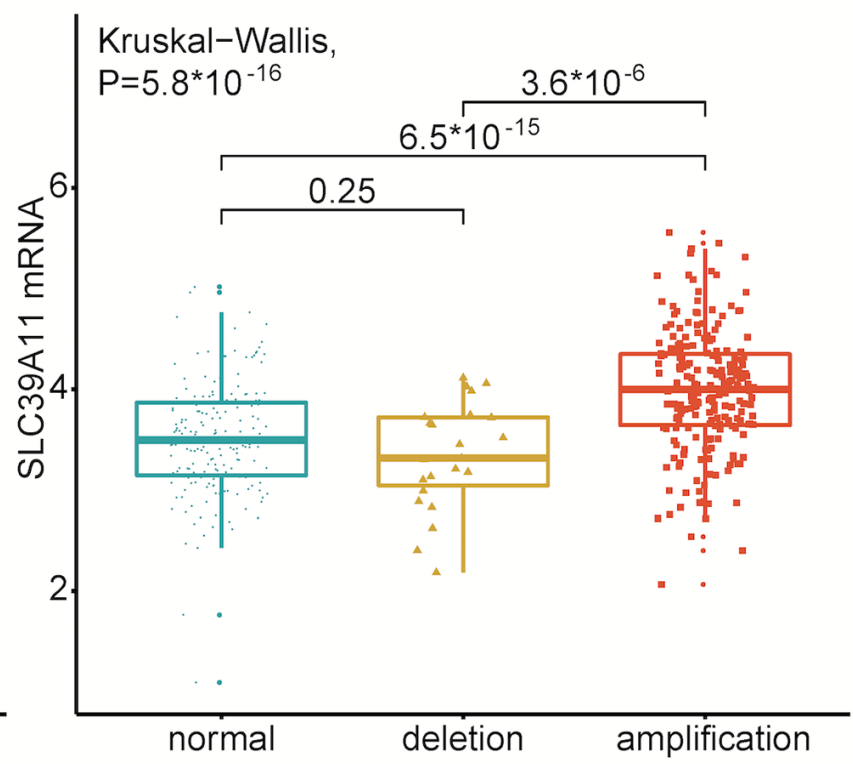

Figure 8. Survival-risk-associated genes in which mRNA level is significantly associated with copy number variation amplification. mRNA expression levels of (A) KCNJ16 and (B) SLC39A11 in different copy number variation status. Multiple comparisons were corrected using Bonferroni's method ( $\alpha$ '=0.0167). $\mathrm{CN}$, copy number. 
Table II. A total of 29 mutant genes are significantly associated with risk scores in patients with non-small cell lung cancer.

\begin{tabular}{|c|c|c|}
\hline Gene name & Location & P-value \\
\hline TP53 & $17 \mathrm{p} 13.1$ & $1.8 \times 10^{-4}$ \\
\hline SMARCA4 & $19 \mathrm{p} 13.2$ & $2.68 \times 10^{-4}$ \\
\hline TRPS1 & $8 \mathrm{q} 23.3$ & $2.07 \times 10^{-3}$ \\
\hline COL22A1 & $8 q 24.23$ & $2.52 \times 10^{-3}$ \\
\hline SCNIA & $2 q 24.3$ & $2.72 \times 10^{-3}$ \\
\hline HYDIN & $16 \mathrm{q} 22.2$ & $2.99 \times 10^{-3}$ \\
\hline$F A M 47 B$ & Xp21.1 & $3.30 \times 10^{-3}$ \\
\hline POTEG & $14 q 11.2$ & $3.39 \times 10^{-3}$ \\
\hline NLRP3 & $1 q 44$ & $3.61 \times 10^{-3}$ \\
\hline$L R P I B$ & $2 q 22.1$ & $3.63 \times 10^{-3}$ \\
\hline KEAPI & $19 \mathrm{p} 13.2$ & $4.05 \times 10^{-3}$ \\
\hline KCNU1 & $8 p 11.23$ & $4.43 \times 10^{-3}$ \\
\hline$F M N 2$ & $1 q 43$ & $5.13 \times 10^{-3}$ \\
\hline MUC16 & $19 \mathrm{p} 13.2$ & $5.22 \times 10^{-3}$ \\
\hline$B S N$ & $3 p 21.31$ & $5.37 \times 10^{-3}$ \\
\hline$R G P D 4$ & $2 q 12.3$ & $5.63 \times 10^{-3}$ \\
\hline PKDIL1 & $7 \mathrm{p} 12.3$ & $5.68 \times 10^{-3}$ \\
\hline$R B P 3$ & $10 q 11.22$ & $5.74 \times 10^{-3}$ \\
\hline$T N N$ & $1 q 25.1$ & $5.80 \times 10^{-3}$ \\
\hline CSMD3 & $8 q 23.3$ & $6.52 \times 10^{-3}$ \\
\hline$A P O B$ & $2 \mathrm{p} 24.1$ & $6.86 \times 10^{-3}$ \\
\hline PCDHB5 & $5 q 31.3$ & $7.85 \times 10^{-3}$ \\
\hline USP9X & Xp11.4 & $7.86 \times 10^{-3}$ \\
\hline$E R B B 4$ & $2 q 34$ & $8.28 \times 10^{-3}$ \\
\hline$Z F H X 4$ & $8 q 21.11$ & $8.68 \times 10^{-3}$ \\
\hline COLIIA1 & $1 \mathrm{p} 21.1$ & $8.78 \times 10^{-3}$ \\
\hline DNAH3 & $16 \mathrm{p} 12.3$ & $9.10 \times 10^{-3}$ \\
\hline MTCL1 & $18 p 11.22$ & $9.61 \times 10^{-3}$ \\
\hline$R Y R 3$ & $15 q 14$ & $9.61 \times 10^{-3}$ \\
\hline
\end{tabular}

copy number variation (-2, -1, 0, 1 and 2$)$ and mutation (0 and 1) was a discrete variable. It's hard to fit a precise prognosis model using discrete variable. Thus, we selected methylation data (continuous variable) were used to construct the survival risk model. The outcome of Cox regression analysis showed that methylation-based survival risk was an independent prediction factor of survival. The survival risk scores for 439 patients with NSCLC were obtained by constructing a methylation-based prognosis model. A total of 35 copy number amplifications regions, 40 copy number deletion regions and 29 mutation genes were identified, which were significantly associated with survival risk.

The present study identified various mutation features in different molecular subtypes. iCluster 2 , iCluster 4 , iCluster 5 and iCluster 6 were characterized by a high frequency of TP53 mutations. Remarkably, TTN also had a high mutation frequency in iCluster 2, iCluster 4, iCluster 5 and iCluster 6 . The distribution of TTN mutations was similar to TP53. Thus, TTN may be an important gene in NSCLC; however, to the best of our knowledge, TTN has not been investigated in previous lung cancer studies.
iCluster 6 had the least favorable outcome for overall survival, while iClusters 1-5 had a more favorable prognosis compared with iCluster 6 . In addition, iCluster 6 had high TTN (66.18\%) and TP53 (66.18\%) mutation rates. Thus, TTN and TP53 mutations may lead to unfavorable survival outcomes for iCluster6.

Based on the methylation datasets, the present study constructed a survival risk model using LASSO regression. Using this model, high survival risk was associated with chromosome 17q24.3 amplification and chromosome 11p15.5 deletion. It has previously been reported that chromosome 17q24.3 is associated with LUAD (39) and with MGMT methylation in the lung (40). Among the amplified genes identified in 17q24.3, AC005152.3, LINC01152, RP11-84E24.2, SOX9-AS1, AC007461.2, SOX9, KCNJ16 and SLC39A11 were the most significant genes in the present study. To the best of our knowledge, RP11-84E24.2 and AC007461.2 have not been identified in previous studies investigating lung cancer. The present study revealed that the deletion of the six survival risk-associated genes (AP2A2, MOB2, MUC5B, MUC6, TOLLIP and TOLLIP-ASI) in $11 \mathrm{p} 15.5$ influenced their mRNA expression levels, whereas the amplification of two survival risk-associated genes (KCNJ16 and $S L C 39 A 11$ ) in 17q24.3 influenced their mRNA expression levels. A previous study reported that the chromosome region at $17 \mathrm{q} 24.3$ was a novel and frequent $\mathrm{LOH}$ region associated with NSCLC (41). The present study showed that the survival risks of patients with amplified genes in 17q24.3 were significantly higher compared with those with deletion or normal $17 \mathrm{q} 24.3$ gene status. It was hypothesized that copy number variation of survival risk-associated genes in 17q24.3 may affect patient prognosis by LOH. The majority of deletion events in $17 q 24.3$ may therefore be due to $\mathrm{LOH}$.

Depletion of SOX9-AS1 and AC005152 leads to a decrease in SOX 9 mRNA and protein expression levels (42). However, the expression levels of LINCO1152 had the opposite trend in contrast to SOX9-AS1, ACO05152 and SOX9 (42). As a gene functioning in cartilage homeostasis, $B M P 3 B$ has tumor-suppressive functions with promoter hypermethylation in lung cancer (43). It has been reported that $S O X 9$ can influence the chondrocyte phenotype through regulating the process of hypoxia (44). Thus, it was considered that SOX9 may influence the expression of $B M P 3 B$, and SOX9-AS1, AC005152 and LINCO1152 may mediate the expression levels of SOX 9 and $B M P 3 P$ indirectly. Therefore, genes that have been identified in chromosome 17q24.3 may serve important roles in lung cancer by regulating the levels of $B M P 3 B$ expression.

The outcome of the present study indicated that deletion of chromosome 11p15.5 may lead to high survival risk for patients with NSCLC. MOB2, AP2A2, MUC6, MUC2, MUC5B, MIR6744, TOLLIP and TOLLIP-AS1 are located within this region and were significantly associated with survival risk. A previous study demonstrated that deletion of TOLLIP can increase the level of mRNA expression of $I L-6$ (45), while a decrease in $M U C 2$ protein expression can increase the level of $I L-6$ expression (46). It has been reported that IL-6 can induce the early response of $M U C 2, M U C 5 B$ and MUC6 (47). IL-6 can activate the STAT3 signaling pathway, which will stimulate the progression of cancer (48). It was proposed that the deletion of genes in 11p15.5 may up-regulate the level of $I L-6$ expression and lead to an increase in survival risk. However, 


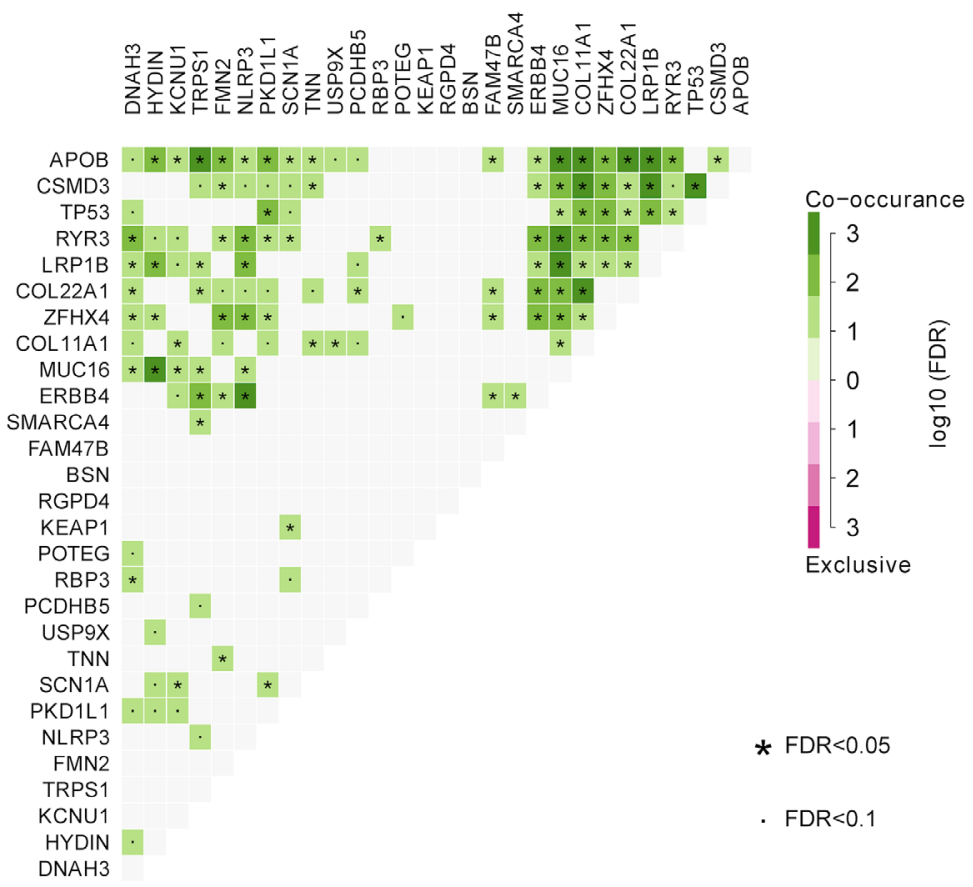

Figure 9. Association between mutant genes and survival risk score. The co-occurance or exclusive associations between 29 mutant genes. The occurrence of CDMD3, ZFHX4 and COL11A1 were simultaneous with TP53 mutation. P-values were corrected using the FDR method.

this association was not found in any of the datasets in the present study.

A total of 29 mutant genes were found to be significantly correlated with survival risk score. Among these genes, 12 genes had significant co-occurrence (MUC16 co-occurred with $H Y D I N, A P O B, R Y R 3$ and $L R P I B ; A P O B$ co-occurred with TRPS1, MUC16, COL11A1, COL22A1 and LRP1B; CSMD3 co-occurred with COL11A1, LRPIB and TP53; ERBB4 co-occurred with NLRP3; COL11A1 co-occurred with $A P O B$, $C S M D 3$ and $C O L 22 A 1$.). A previous study demonstrated that overexpression of $M U C 16$ can promote tumor cell proliferation and migration by activating the JAK2/STAT3/GR axis (49). Overexpression of TRPS1 leads to multi-drug resistance by inducing $M G M T$ transcription in lung cancer (50). It was reported that $C O L 11 A 1$ can promote the proliferation, migration and invasion of NSCLC cell lines in vitro (51). Qiu et al (52) reported that mutation of CSMD3 will lead resistance to etoposide in small-cell lung cancer. Teng et al (53) reported that activation of NLRP3 may induce pyroptosis in NSCLC. The second-generation inhibitor of $E R B B 4$ has passed the phase III clinical trial (54). It was reported that mutation of $L R P I B$ correlated with better response of immune therapy and higher tumor mutation load (55). However, the other co-occurrence genes we identified lacked NSCLC-associated research. The co-occurrence associations of genes we identified may provide direction for further clinical and therapy studies.

In conclusion, integrated molecular subtypes of NSCLC were identified by integrated analytic approaches. The chromosome regions $17 \mathrm{q} 24.3$ and 11 p15.5 were identified as survival-associated copy number variation regions, while a total of 29 mutant genes were found to be significantly associated with survival. Through assessing the level of methylation sites in the present model, the survival risk of patients was predicted and this may be beneficial to estimate the prognosis of patients with lung cancer. In addition, the $\mathrm{CpG}$ sites identified in the present study require further investigation to understand their functions in lung cancer. Genes corresponding to these $\mathrm{CpG}$ sites may serve as novel therapeutic targets for lung cancer treatment in the future.

\section{Acknowledgements}

Not applicable.

\section{Funding}

No funding was received.

\section{Availability of data and materials}

The datasets used and/or analyzed during the present study are available from the corresponding author upon reasonable request.

\section{Authors' contributions}

ML and SQ designed the study. HS, FS, XM, YD, CS and HZ collected and analyzed the data. JJ downloaded TCGA data. ML, SQ and JJ performed analysis of data. HS, FS and XM reviewed the results and interpreted the data. ML and SQ wrote the manuscript. HS, YD, CS and HZ gave final approval of the version to be published. All authors read and approved the manuscript and agreed to be accountable for all aspects of the research in ensuring that the accuracy or integrity of any part of the work are appropriately investigated and resolved.

\section{Ethics approval and consent to participate}

Not applicable. 


\section{Patient consent for publication}

Not applicable.

\section{Competing interests}

The authors declare that they have no competing interests.

\section{References}

1. Bray F, Ferlay J, Soerjomataram I, Siegel RL, Torre LA and Jemal A: Global cancer statistics 2018: GLOBOCAN estimates of incidence and mortality worldwide for 36 cancers in 185 countries. CA Cancer J Clin 68: 394-424, 2018.

2. Molina JR, Yang P, Cassivi SD, Schild SE and Adjei AA: Non-small cell lung cancer: Epidemiology, risk factors, treatment, and survivorship. Mayo Clinic Oroceedings 83: 584-594, 2008.

3. Miller KD, Siegel RL, Lin CC, Mariotto AB, Kramer JL, Rowland JH, Stein KD, Alteri R and Jemal A: Cancer treatment and survivorship statistics, 2016. CA Cancer J Clin 66: 271-289, 2016.

4. Chen Z, Fillmore CM, Hammerman PS, Kim CF and Wong KK: Non-small-cell lung cancers: A heterogeneous set of diseases. Nat Rev Cancer 14: 535-546, 2014.

5. Del Prete C and Azzoli CG: Non-small cell lung cancer in the Era of personalized medicine: Molecular tests that matter. R I Med J (2013) 103: 28-32, 2020

6. Muller IB, de Langen AJ, Giovannetti E and Peters GJ: Anaplastic lymphoma kinase inhibition in metastatic non-small cell lung cancer: Clinical impact of alectinib. Onco Targets Ther 10 4535-4541, 2017.

7. Bergethon K, Shaw AT, Ou SH, Katayama R, Lovly CM, McDonald NT, Massion PP, Siwak-Tapp C, Gonzalez A, Fang R, et al: ROS1 rearrangements define a unique molecular class of lung cancers. J Clin Oncol 30: 863-870, 2012

8. Paez JG, Janne PA, Lee JC, Tracy S, Greulich H, Gabriel S, Herman P, Kaye FJ, Lindeman N, Boggon TJ, et al: EGFR mutations in lung cancer: Correlation with clinical response to gefitinib therapy. Science 304: 1497-1500, 2004

9. Comprehensive molecular characterization of human colon and rectal cancer. Nature 487: 330-337, 2012

10. Ronglai S, Olshen AB and Marc L: Integrative clustering of multiple genomic data types using a joint latent variable model with application to breast and lung cancer subtype analysis. Bioinformatics 25: 2906, 2009.

11. Cancer Genome Atlas Research Network. Electronic address: wheeler@bcm.edu; Cancer Genome Atlas Research Network: Comprehensive and integrative genomic characterization of hepatocellular carcinoma. Cell 169: 1327-1341.e23, 2017.

12. Cancer Genome Atlas Research Network: Comprehensive molecular profiling of lung adenocarcinoma. Nature 511: 543-550, 2014

13. Yue QY,Zhao W, Tan Y, Deng XL and Zhang YH: PLCE1 inhibits apoptosis of non-small cell lung cancer via promoting PTEN methylation. Eur Rev Med Pharmacol Sci 23: 6211-6216, 2019.

14. Shahabi S, Kumaran V, Castillo J, Cong Z, Nandagopal G Mullen DJ, Alvarado A, Correa MR, Saizan A, Goel R, et al: LINC00261 Is an epigenetically regulated tumor suppressor essential for activation of the DNA damage response. Cancer Res 79: 3050-3062, 2019.

15. Yun J, Mi HP, Dong JS, Nam KT, Moon DB, Ju JH, Hwang OK, Choi JS, Kim TH, Jung YS, et al: IL-32 gamma reduces lung tumor development through upregulation of TIMP-3 overexpression and hypomethylation. Cell Death Dis 9: 306, 2018.

16. Kang X, Kong F, Huang K, Li L, Li Z, Wang X, Zhang W and Wu X: LncRNA MIR210HG promotes proliferation and invasion of non-small cell lung cancer by upregulating methylation of CACNA2D2 promoter via binding to DNMT1. Onco Targets Ther 12: 3779-3790, 2019.

17. Wang Y, Zhang Q, Gao Z, Xin S, Zhao Y, Zhang K, Shi R and Bao X: A novel 4-gene signature for overall survival prediction in lung adenocarcinoma patients with lymph node metastasis. Cancer Cell Int 19: 100, 2019.

18. Liu C, Li Y, Wei M, Zhao L, Yu Y and Li G: Identification of a novel glycolysis-related gene signature that can predict the survival of patients with lung adenocarcinoma. Cell Cycle 18 : $568-579,2019$
19. Songyang Y, Zhu W, Liu C, Li LL, Hu W, Zhou Q, Zhang H, $\mathrm{Li} \mathrm{W}$ and Li D: Large-scale gene expression analysis reveals robust gene signatures for prognosis prediction in lung adenocarcinoma. PeerJ 7: e6980, 2019.

20. R Core Team (2019). R: A language and environment for statistical computing. R Foundation for Statistical Computing, Vienna, Austria. URL https://www.R-project.org/.

21. Colaprico A, Silva TC, Olsen C, Garofano L, Cava C, Garolini D, Sabedot TS, Malta TM, Pagnotta SM, Castiglioni I, et al: TCGAbiolinks: An R/Bioconductor package for integrative analysis of TCGA data. Nucleic Acids Res 44: e71, 2016.

22. Mo Q, Wang S, Seshan VE, Olshen AB, Schultz N, Sander C, Powers RS, Ladanyi M and Shen R: Pattern discovery and cancer gene identification in integrated cancer genomic data. Proc Natl Acad Sci USA 110: 4245-4250, 2013.

23. Xu T, Duy LT, Liu L, Su N, Wang R, Sun B, Colaprico A, Bontempi G and Li J: CancerSubtypes: An R/Bioconductor package for molecular cancer subtype identification, validation, and visualization. Bioinformatics 33: 3131-3133, 2017.

24. Sadanori K and Genshiro K: Bayesian information Criteria. In: Information Criteria and Statistical Modeling. Springer Series in Statistics. Springer, New York, NY, 2008.

25. Kassambara A and Kosinski M: Survminer: Drawing Survival Curves using 'ggplot2', 2018.

26. Dardis C: survMisc: Miscellaneous functions for survival data, 2018.

27. Bolstad B: PreprocessCore: A collection of pre-processing functions, 2018.

28. Therneau TM: A package for survival analysis in S, 2015.

29. Simon N, Friedman J, Hastie T and Tibshirani R: Regularization paths for Cox's proportional hazards model via coordinate descent. J Stat Softw 39: 1-13, 2011.

30. Frank E Harrell Jr, with contributions from Charles Dupont and many others. Hmisc: Harrell Miscellaneous. R package version 4.4-0, 2020.

31. Caesar-Johnson SJ, Demchok JA, Felau I, et al: The immune landscape of cancer. Immunity 81: 105, 2018.

32. Taylor AM, Shih J, Ha G, Gao GF, Zhang X, Berger AC, Schumacher SE, Wang C, Hu H, Liu J, et al: Genomic and functional approaches to understanding cancer aneuploidy. Cancer Cell 33: 676-689.e3, 2018

33. Cerami E, Gao J, Dogrusoz U, Gross BE, Sumer SO, Aksoy BA, Jacobsen A, Byrne CJ, Heuer ML, Larsson E, et al: The cBio cancer genomics portal: An open platform for exploring multidimensional cancer genomics data. Cancer Discov 2: 401-404, 2012.

34. Gao J, Aksoy BA, Dogrusoz U, Dresdner G, Gross B, Sumer SO Sun Y, Jacobsen A, Sinha R, Larsson E, et al: Integrative analysis of complex cancer genomics and clinical profiles using the cBioPortal. Sci Signal 6: pl1, 2013.

35. Kassambara A: Ggpubr: 'Ggplot2' Based Publication Ready Plots, 2018.

36. Mayakonda A and Koeffler HP: Maftools: Efficient analysis, visualization and summarization of MAF files from large-scale cohort based cancer studies. bioRxiv: 052662, 2016 doi: https:// doi.org/10.1101/052662.

37. Chun YS, Pawlik TM and Vauthey JN: 8th Edition of the AJCC cancer staging manual: Pancreas and hepatobiliary cancers. Ann Surg Oncol 25: 845-847, 2018.

38. Cancer Genome Atlas Research Network: Comprehensive molecular profiling of lung adenocarcinoma. Nature 511: 543-550, 2014.

39. Wei JS, Matsuo K, Hsiung CA, Shiraishi K, Song M, Kim HN, Wong MP, Hong YC, Hosgood HD III, Wang Z, et al: Association between GWAS-identified lung adenocarcinoma susceptibility loci and EGFR mutations in never-smoking Asian women, and comparison with findings from Western populations. Hum Mol Genet 26: 454-465, 2016.

40. Leng S, Wu G, Collins LB, Thomas CL, Tellez CS, Jauregui AR, Picchi MA, Zhang X, Juri DE, Desai D, et al: Implication of a chromosome $15 \mathrm{q} 15.2$ locus in regulating UBR1 and predisposing smokers to MGMT methylation in lung. Cancer Res 75: 3108-3117, 2015.

41. Tseng RC, Chang JW, Hsien FJ, Chang YH, Hsiao CF, Chen JT, Chen CY, Jou YS and Wang YC: Genomewide loss of heterozygosity and its clinical associations in non small cell lung cancer. Int J Cancer 117: 241-247, 2005.

42. Barter MJ, Gomez R, Hyatt S, Cheung K, Skelton AJ, Xu Y, Clark IM and Young DA: The long non-coding RNA ROCR contributes to SOX9 expression and chondrogenic differentiation of human mesenchymal stem cells. Development 144: 4510-4521, 2017.

43. Dai Z, Popkie AP, Zhu WG, Timmers CD, Raval A, Tannehill-Gregg S, Morrison CD, Auer H, Kratzke RA, Niehans G, et al: Bone morphogenetic protein 3B silencing in non-small-cell lung cancer. Oncogene 23: 3521-3529, 2004. 
44. Lafont J, Talma S, Hopfgarten C and Murphy CL: Hypoxia promotes the differentiated human articular chondrocyte phenotype through SOX9-dependent and -independent pathways. J Biol Chem 283: 4778-4786, 2008.

45. Humbert-Claude M, Duc D, Dwir D, Thieren L, Sandström von Tobel J, Begka C, Legueux F, Velin D, Maillard MH, Do KQ, et al: Tollip, an early regulator of the acute inflammatory response in the substantia nigra. J Neuroinflammation 13: 303, 2016.

46. Hsu HP, Lai MD, Lee JC, Yen MC, Weng TY, Chen WC, Fang JH and Chen YL: Mucin 2 silencing promotes colon cancer metastasis through interleukin-6 signaling. Sci Rep 7: 5823, 2017.

47. Enss ML, Cornberg M, Wagner S, Gebert A, Henrichs M, Eisenblätter R, Beil W, Kownatzki R and Hedrich $\mathrm{HJ}$ : Proinflammatory cytokines trigger MUC gene expression and mucin release in the intestinal cancer cell line LS180. Inflamm Res 49: 162-169, 2000.

48. Rebouissou S, Amessou M, Couchy G, Poussin K, Imbeaud S, Pilati C, Izard T, Balabaud C, Bioulac-Sage P and Zucman-Rossi J: Frequent in-frame somatic deletions activate gp130 in inflammatory hepatocellular tumours. Nature 457: 200-204, 2008.

49. Lakshmanan I, Salfity S, Seshacharyulu P, Rachagani S, Thomas A, Das S, Majhi PD, Nimmakayala RK, Vengoji R Lele SM, et al: MUC16 regulates TSPYL5 for lung cancer cell growth and chemoresistance by suppressing p53. Clin Cancer Res 23: 3906-3917, 2017.

50. Liu H, Liao Y, Tang M, Wu T, Tan D, Zhang S and Wang H: Trps1 is associated with the multidrug resistance of lung cancer cell by regulating MGMT gene expression. Cancer Med 7: 1921-1932, 2018.
51. Shen L, Yang M, Lin Q, Zhang Z, Zhu B and Miao C: COL11A1 is overexpressed in recurrent non-small cell lung cancer and promotes cell proliferation, migration, invasion and drug resistance. Oncol Rep 36: 877-885, 2016.

52. Qiu Z, Lin A, Li K, Lin W, Wang Q, Wei T, Zhu W, Luo P and Zhang J: A novel mutation panel for predicting etoposide resistance in small-cell lung cancer. Drug Des Devel Ther 13: 2021-2041, 2019

53. Teng JF, Mei QB, Zhou XG, Tang Y, Xiong R, Qiu WQ, Pan R, Law BY, Wong VK, Yu CL, et al: Polyphyllin VI induces caspase-1-mediated pyroptosis via the induction of ROS/NF- $\kappa \mathrm{B} /$ NLRP3/GSDMD signal axis in non-small cell lung cancer. Cancers 12: 193, 2020.

54. Sun $\mathrm{H}$ and Wu YL: Dacomitinib in non-small-cell lung cancer: A comprehensive review for clinical application. Future Oncol 15: 2769-2777, 2019.

55. Chen H, Chong W, Wu Q, Yao Y, Mao M and Wang X: Corrigendum: Association of LRP1B mutation with tumor mutation burden and outcomes in melanoma and non-small cell lung cancer patients treated with immune check-point blockades. Front Immunol 10: 1523, 2019.

This work is licensed under a Creative Commons Attribution-NonCommercial-NoDerivatives 4.0 International (CC BY-NC-ND 4.0) License. 